EPJ Web of Conferences 47, 06008 (2013)

DOI: $10.1051 /$ epjconf/20134706008

(C) Owned by the authors, published by EDP Sciences, 2013

\title{
White dwarf planets
}

\author{
Alexander J. Mustill ${ }^{1, a}$, Eva Villaver ${ }^{1}$, Dimitri Veras ${ }^{2}$, Amy Bonsor $^{3}$ \\ and Mark C. Wyatt ${ }^{2}$ \\ ${ }^{1}$ Departamento de Física Teórica, Universidad Autónoma de Madrid, Cantoblanco, \\ 28049 Madrid, España \\ 2 Institute of Astronomy, University of Cambridge, Madingley Road, Cambridge CB3 OHA, UK \\ 3 UJF-Grenoble 1 / CNRS-INSU, Institut de Planétologie et d'Astrophysique de Grenoble \\ (IPAG), UMR 5274, BP. 53, 38041 Grenoble Cedex 9, France
}

\begin{abstract}
The recognition that planets may survive the late stages of stellar evolution, and the prospects for finding them around White Dwarfs, are growing. We discuss two aspects governing planetary survival through stellar evolution to the White Dwarf stage. First we discuss the case of a single planet, and its survival under the effects of stellar mass loss, radius expansion, and tidal orbital decay as the star evolves along the Asymptotic Giant Branch. We show that, for stars initially of $1-5 \mathrm{M}_{\odot}$, any planets within about $1-5 \mathrm{AU}$ will be engulfed, this distance depending on the stellar and planet masses and the planet's eccentricity. Planets engulfed by the star's envelope are unlikely to survive. Hence, planets surviving the Asymptotic Giant Branch phase will probably be found beyond $\sim 2 \mathrm{AU}$ for a $1 \mathrm{M}_{\odot}$ progenitor and $\sim 10 \mathrm{AU}$ for a $5 \mathrm{M}_{\odot}$ progenitor. We then discuss the evolution of two-planet systems around evolving stars. As stars lose mass, planet-planet interactions become stronger, and many systems stable on the Main Sequence become destabilised following evolution of the primary. The outcome of such instabilities is typically the ejection of one planet, with the survivor being left on an eccentric orbit. These eccentric planets could in turn be responsible for feeding planetesimals into the neighbourhood of White Dwarfs, causing observed pollution and circumstellar discs.
\end{abstract}

\section{INTRODUCTION}

Much interest is now focussing on planetary systems around evolved stars. Radial-velocity surveys have discovered several dozen planets orbiting giant stars [see 10, for a recent compilation]. The existence of White Dwarfs with polluted atmospheres or circumstellar discs strongly suggests the existence of planets and/or planetesimals to deliver the heavy elements to the star [6, 8, 9, 23]. Searches have been made for planets orbiting White Dwarfs [e.g., 5, 15], although so far there are no secure detections. Claims have also been made of detections of planets around evolved binaries [e.g., 14], although such claims often do not survive detailed scrutiny [e.g., 21].

Concomitant with such observational efforts is a drive to gain a theoretical understanding of the processes acting on planetary systems as stars evolve. The expansion of the stellar radius on the Red Giant Branch (RGB) and Asymptotic Giant Branch (AGB) can strengthen the tidal forces pulling planets into the star and result in their engulfment and destruction [e.g., 20]. The greatly increased stellar luminosity, particularly at the AGB tip, can cause significant evaporation of planetary atmospheres [19]. The expulsion of material from the star on the AGB causes the expansion of planetary orbits [12], while drag from the expelled material can cause the orbits of planetesimals to shrink [4]. The reduction of

\footnotetext{
ae-mail: alex.mustill@uam.es
}

This is an Open Access article distributed under the terms of the Creative Commons Attribution License 2.0, which permits unrestricted use, distribution, and reproduction in any medium, provided the original work is properly cited. 


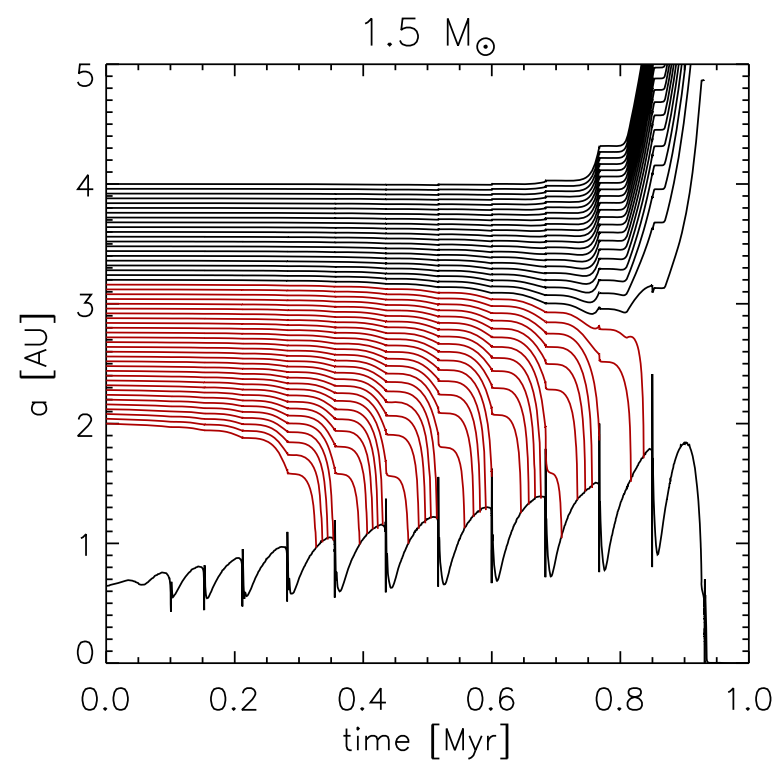

Figure 1. Semi-major axis evolution of Jovian-mass planets on circular orbits around a $1.5 \mathrm{M}_{\odot}$ AGB star The lower line shows the evolution of the stellar radius. Red lines indicate planets that are engulfed in the stellar envelope, black those that avoid engulfment. Originally published as Fig. 2 of "Foretellings of Ragnarök: World-engulfing Asymptotic Giants and the Inheritance of White Dwarfs", Mustill, A. J. \& Villaver, E., The Astrophysical Journal, 761, 2, 121. Reproduced by permission of the AAS.

the stellar mass also increases the planet:star mass ratios, significantly altering systems' dynamics and potentially triggering instability $[2,3]$.

In this contribution we summarise our recent work on the survival of planets around AGB stars, including the effects of stellar mass loss, radius changes and tidal forces [13]. We then summarise our work on the dynamics of two-planet systems around evolving stars [18].

\section{PLANETARY SURVIVAL AROUND AGB STARS}

During the AGB stage at the end of a star's nuclear-burning life, the stellar radius expands to very large values of several au, and the star loses a significant fraction of its mass, ranging from $\sim 50 \%$ for a $1 \mathrm{M}_{\odot}$ star to $\sim 80 \%$ for a $5 \mathrm{M}_{\odot}$ star. As the star loses mass, the orbital radii of all bodies must expand to conserve angular momentum [see 17, for a detailed discussion]. However, planets within a few au of the star feel the effects of very strong tidal forces, due to the large stellar radius and the efficient tidal dissipation in the deep convective envelope [20]. These tidal forces draw the planets towards and even into the envelope where they will likely be destroyed. Hence, the survival of planets around AGB stars is determined by the stellar mass loss casuing orbital expansion and the tidal forces dragging the planets in.

We studied the behaviour of planets of Earth, Neptune and Jupiter mass, around AGB stars of $1-5 \mathrm{M}_{\odot}$. We used the stellar models of [16], which accurately treat the strong fluctuations of stellar radius during the thermal pulses, and include a realistic mass loss prescription. To model the tidal evolution we adopted the theory of [22], in which tidal energy is dissipated by turbulent convection in the large envelope of the giant star. We considered planets with eccentricities of 0 and 0.1 .

A typical evolution of a suite of planets is shown in Fig. 1. Jupiter-mass planets are started on circular orbits around a $1.5 \mathrm{M}_{\odot}$ star. Those within around 3 au are drawn into the stellar envelope by tidal forces, while those on wider orbits escape. The critical radius at which planets survive for a range of stellar and 


\section{Hot Planets and Cool Stars}
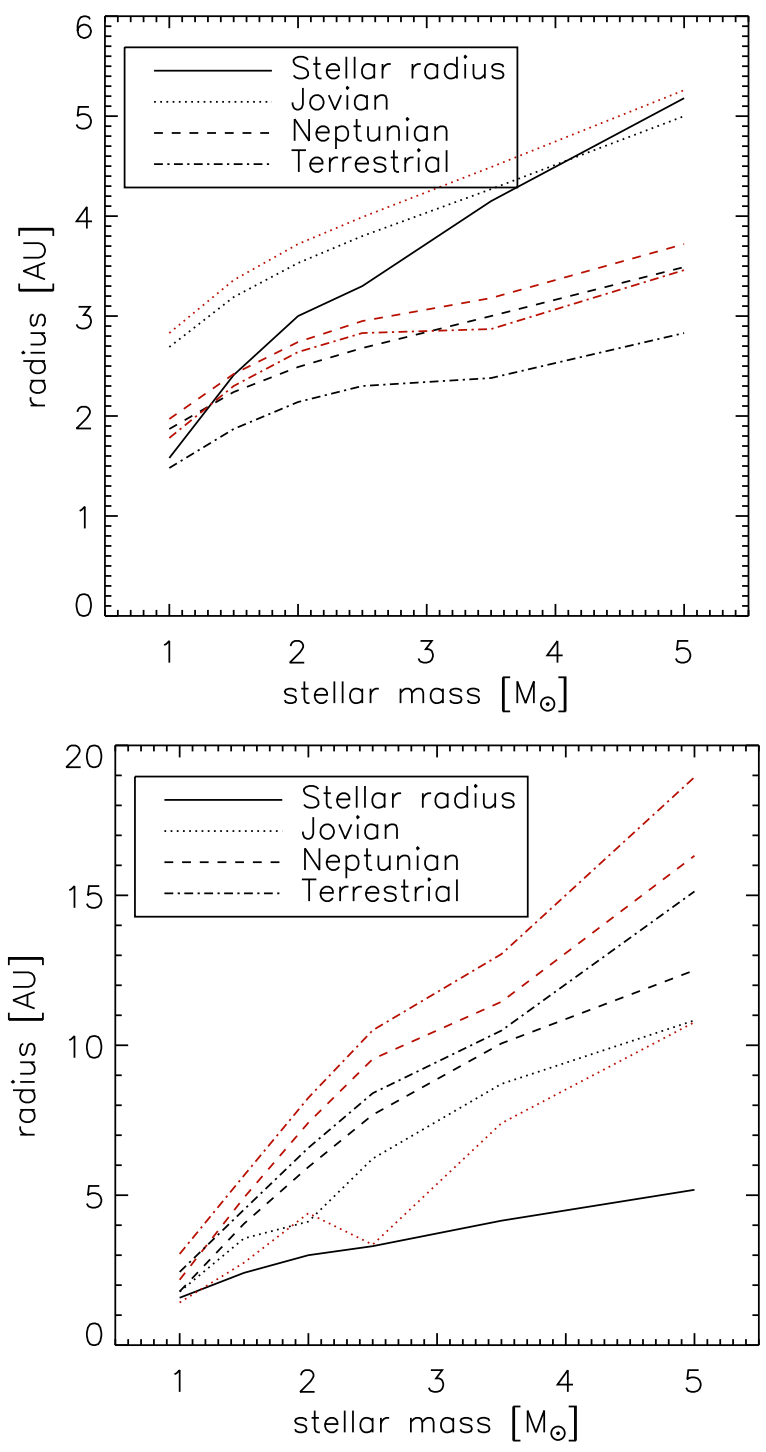

Figure 2. Top: critical radius within which planets will be engulfed by the envelope of an AGB star. Solid line marks the maximum stellar radius. Dotted, dashed and dot-dashed show different planet masses. Black lines mark planets on initialy circular orbits, red planets with an initial eccentricity of 0.1 . Originally published as Fig. 7 of Mustill \& Villaver, op. cit. Reproduced by permission of the AAS. Bottom: final radius of closest surviving planet at the end of the AGB. Line styles are as in top panel. Originally published as Fig. 10 of Mustill \& Villaver, op. cit. Reproduced by permission of the AAS.

planet masses is shown in the upper panel of Fig. 2. This is larger for higher-mass stars, as these stars attain a higher maximum radius on the AGB, and for higher-mass planets, since these raise stronger tides on the host star and hence are subjected to stronger tidal forces. It is also larger for eccentric planets, for high planet masses because tidal forces are strengthened by an eccentric orbit, and in the low mass case because the planets' lower pericentres take them closer to the stellar envelope.

The Earth-mass planets feel almost no effect from tidal forces at all, and their evolution is entirely governed by mass-loss induced expansion. In summary, planets beginning the AGB 
within $1.5-5 \mathrm{au}$ of their host star, depending on planet and stellar properties, will find themselves engulfed.

We also calculated the closest radius at which surviving planets may be found. Due to orbital expansion during mass loss, this is typically much larger than the radius of the most distant engulfed planet. In the absence of tidal forces, the former would be exactly the latter scaled up by the expansion factor from adiabatic mass loss, although for more massive planets the tidal drag reduces the value somewhat. These minimum radii of survivors are shown in the lower panel of Fig. 2. Values range from around 2 au for $1 \mathrm{M}_{\odot}$ stars to $10-20$ au for $5 \mathrm{M}_{\odot}$ stars, depending on planet mass. Hence, White Dwarfs may be surrounded by a large volume several au in radius cleared of all planetary bodies.

\section{PLANET-PLANET SCATTERING AND STELLAR EVOLUTION}

The loss of stellar mass on the AGB, as well as causing the planets' orbits to expand, increases the planet:star mass ratios, and hence planet-planet interactions of all kinds become more significant following mass loss. Many planets orbiting Main-Sequence stars are in closely-packed systems close to the limit of stability, and when planet-planet interactions in such systems strengthen following mass loss the systems may be destabilised [3]. Such instabilities may deliver either planets themselves, or more indirectly smaller asteroids or comets, to the neighbourhood of the WD, potentially creating circumstellar discs and atmospheric pollution [e.g., 2, 3]. In [18] we revisited the dynamics of twoplanet systems orbiting evolving stars. We followed the dynamics of such systems over the whole stellar lifetime onto the WD phase, to an age of 5 Gyr. Unfortunately, as the Main-Sequence lifetime of stars is such a strong function of mass, to integrate a significnt number of systems we were restricted to relatively large stellar masses, of $3-8 \mathrm{M}_{\odot}$, the greatest value being chosen as this is the greatest progenitor mass that produces a WD. Our fiducial systems were composed of two planets of Jovian mass, the inner initially orbiting at 10 au and the outer slightly further out, both in moderately eccentric orbits $(e=0.1)$.

The stability of three-body systems can to some extent be characterised analytically, through the Hill stability criterion. If the semi-major axis ratio of two planets is greater than some critical value, determined by the planets' masses, eccentricities and inclinations, then the orbits of the two planets can never intersect (an instability known as Hill instability). This does not preclude that one planet may be driven into the star or ejected from the system (Lagrange instability). However, if the planets' semimajor axis ratio is less than this critical value, then nothing can be said about either Hill or Lagrange instability. Hence, N-body integrations are required to properly determine the stability of systems, although in practice under certain conditions the Hill criterion proves useful [e.g., 1]. In the context of stelar evolution, the critical boundary for Hill stability moves outwards as the star loses mass, placing more systems in the potentially unstable region [3].

Our full-lifetime integrations of the two-planet systems show that, as a population, they experience three phases of instability (see Fig. 3). (1) Closely-spaced systems, within the analytical Hill stability limit, are unstable on short time-scales of typically $\lesssim 10^{6}$ years. (2) More widely-spaced systems, just beyond the analytical Hill stability limit, may be unstable on longer time-scales of $\sim 10^{8}$ years. (3) More widely-spaced systems still, and any surviving closer ones, may be destabilised after the star ends its Main-Sequence lifetime, typically after becoming a White Dwarf.

The outcome of instability around giant and White Dwarf stars is overwhelmingly the ejection of one of the planets, leaving the other on a highly eccentric orbit. Survivors around White Dwarfs, despite having semi-major axes of several 10s of AU, may yet have sufficient eccentricities to carry them within the maximum radius of the star on the AGB, a region that should not be populated by survivors of single planet systems [13]. Nevertheless, direct pollution of a White Dwarf by a planet is extremely rare, occurring in less than $1 \%$ of all simulated Jovian systems, and in none of an additional suite of 632 systems of two Earth-mass planets. This suggests that direct pollution of White Dwarfs by planets is rare. However, the scattering events and subsequent highly eccentric planets in unstable systems will 
Instability Times for $M_{\star}(t=0)=3 M_{\odot}$

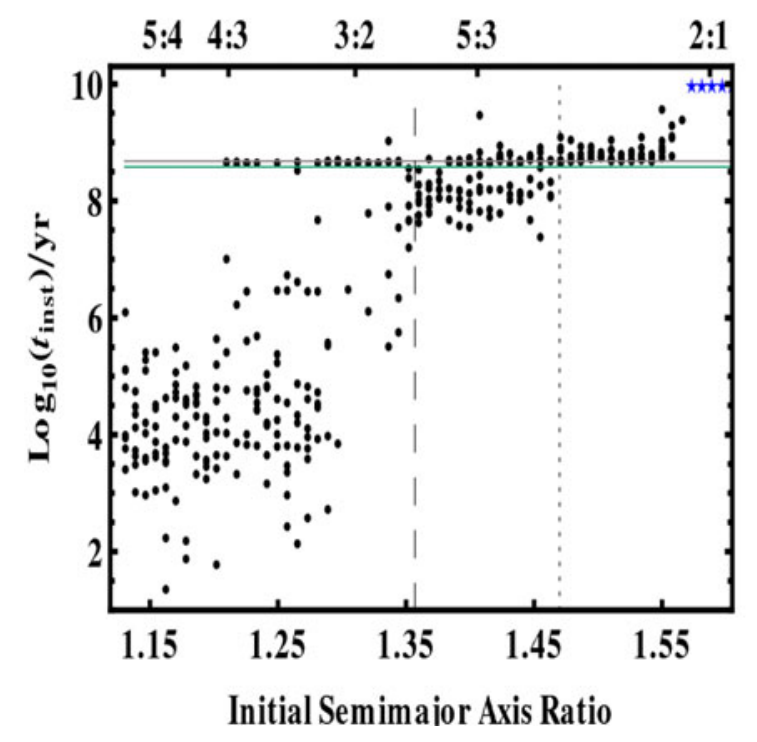

Figure 3. Lifetimes of systems of two Jupiter-mass planets orbiting a $3 \mathrm{M}_{\odot}$ star. Horizontal lines mark transitions in the star's evolutionary state from the Main Sequence to a White Dwarf. Vertical lines mark the Hill stability boundaries before and after post-Main Sequence mass loss. From [18].

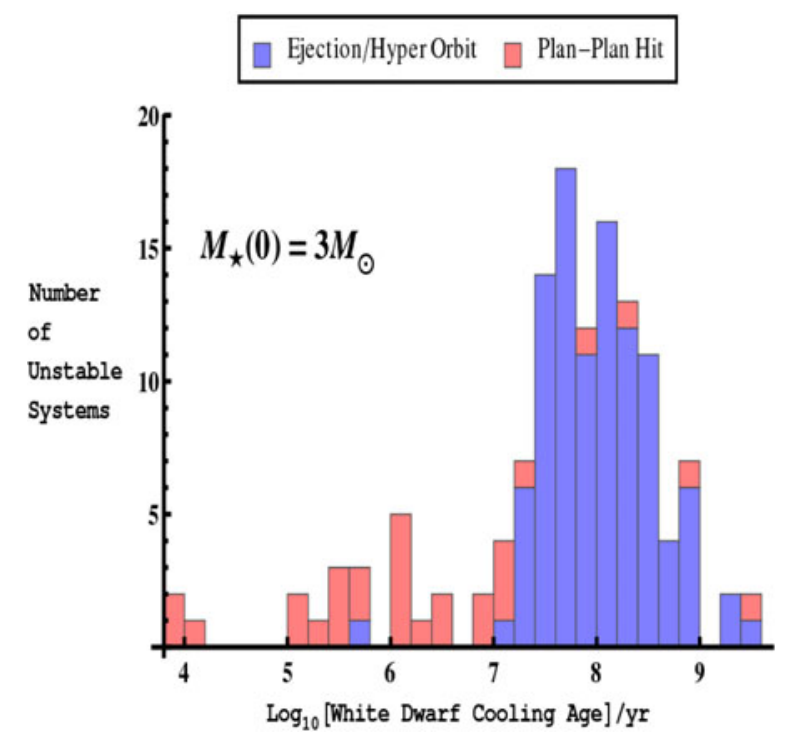

Figure 4. Cooling ages at which instability occurs around simulated White Dwarfs with $3 \mathrm{M}_{\odot}$ progenitors. From [18].

destabilise many previously stable cometary or asteroidal bodies, making such instabilities a promising trigger of White Dwarf pollution by indirect means. The cooling ages of the White Dwarfs around which the instabilities occur are shown in Fig. 4. These cluster around $10^{8}$ years, similar to the cooling ages of observed polluted White Dwarfs [7, 11]. 
EPJ Web of Conferences

\section{CONCLUSIONS}

We have considered two aspects of the post-Main Sequence evolution of planetary systems: the effects of tides and stellar mass loss on single planets within a few AU of their host AGB stars, and the effects of three-body dynamics and stellar mass loss on systems of two planets at 10 AU over the whole stellar lifetime. Our results in [13] show that AGB stars should destroy all planets initially within a few au, and survivors should not be found within $\sim 2$ au of a White Dwarf with a $1 \mathrm{M}_{\odot}$ progenitor and $\sim 10$ au of a White Dwarf with a $5 \mathrm{M}_{\odot}$ progenitor. In [18] we showed that many two-planet systems that are stable on the MS become unstable when the star becomes a WD, and that these systems could plausibly pollute the WDs indirectly by destabilising planetesimals following the planetary instability itself.

AJM thanks the conference organisers for financial support.

\section{References}

[1] Barnes, R. \& Greenberg, R., ApJ, 647, L163 (2006)

[2] Bonsor, A., Mustill, A. J., \& Wyatt, M. C., MNRAS 414, 930 (2011)

[3] Debes, J. H. \& Sigurdsson, S., ApJ, 572, 556 (2002)

[4] Dong, R., Wang, Y., Lin, D. N. C., \& Liu, X. W., ApJ, 715, 1036 (2010)

[5] Faedi, F., West, R. G., Burleigh, M. R., Goad, M. R., \& Hebb, L., MNRAS, 410, 899 (2011)

[6] Farihi, J., Jura., M, \& Zuckerman, B., ApJ, 694, 805 (2009)

[7] Farihi, J., Gänsicke, B. T., Wyatt, M. C., et al., MNRAS, 424, 464 (2012)

[8] Gänsicke, B. T., Koester, D., Farihi, J., et al., MNRAS, 424, 333 (2012)

[9] Gänsicke, B. T., Marsh, T. R., Southworth, J., Rebassa-Mansergas, A., Science, 314, 1908 (2006)

[10] Gettel, S., Wolszczan, A., Niedzielski, A., et al., ApJ, 745, 28 (2012)

[11] Girven, J., Brinkworth, C. S., Farihi, J., et al., ApJ, 749, 154 (2012)

[12] Hadjidemetriou, J. D., Icarus, 2, 440 (1963)

[13] Mustill, A. J. \& Villaver, E., ApJ, 761, 121 (2012)

[14] Qian, S. B., Liu, L., Liao, W. P., et al., MNRAS, 414, L16 (2011)

[15] Steele, P. R., Burleigh, M. R., Dobie, P. D., et al., MNRAS, 416, 2768 (2011)

[16] Vassiliadis, E. \& Wood, P. R., ApJ, 413, 641 (1993)

[17] Veras, D., Wyatt, M. C., Mustill, A. J., Bonsor, A., \& Eldridge, J. J., MNRAS, 417, 2104 (2011)

[18] Veras, D., Mustill, A. J., Bonsor, A., \& Wyatt, M. C., MNRAS, submitted (2013)

[19] Villaver, E. \& Livio, M., ApJ, 661, 1192 (2007)

[20] Villaver, E. \& Livio, M., ApJ, 705, L81 (2009)

[21] Wittenmyer, R. A., Horner, J., Marshall, J. P., Butters, O. W., Tinney, C. G., MNRAS, 419, 3258 (2012)

[22] Zahn, J.-P., A\&A, 57, 383 (1977)

[23] Zuckerman, B., Koester, D., Reid, I. N., \& Hünsch, M., ApJ, 596, 477 (2003) 